\title{
ARTICLE \\ Comparative strategies for stem cell biodistribution in a preclinical study
}

Fang Wang ${ }^{1,2}$, Zhe Wang ${ }^{1,3}$, Fen Wang ${ }^{4}$, Kelly Dong ${ }^{1,2}$, Jing Zhang ${ }^{1,3}$, Yun-juan Sun ${ }^{2}$, Chun-feng Liu ${ }^{4,5}$, Mei-jie Xing ${ }^{2}$, Xue Cheng ${ }^{2}$, Su Wei ${ }^{2}$, Jia-wei Zheng ${ }^{6}$, Xiong-fei Zhao ${ }^{6}$, Xiao-ming Wang ${ }^{6}$, Jie Fu ${ }^{1,3}$ and Hai-feng Song ${ }^{1,3}$

Stem cell therapy represents the potential alternative effective strategy for some diseases that lack effective treatment currently. Correspondingly, it is crucial to establish high-sensitive and reliable quantification assay for tracing exogenous cell migration. In the present study, we first used both bioluminescence imaging (BLI) indirect labeling (human norepinephrine transporter-luciferase reporter system) and ${ }^{89}$ zirconium $\left({ }^{89} \mathrm{Zr}\right.$ )-hNSCs direct labeling combined with positron emission tomography/computer tomography (PET/CT) system for tracking human neural stem cells (hNSCs) migration into the brain via nasal administration in preclinical study. But the above two methods failed to give the biodistribution profile due to their low sensitivity. Considering its superior sensitivity and absolute quantitation capability, we developed and validated the droplet digital PCR (ddPCR) targeting species-specific gene in frozen and paraffin sections, slices, and whole blood with the sensitivity of 100-200 hNSCs. Accurate and high throughput quantification could be performed using ddPCR with the coefficient of variation (CVs) of lower quality control (LQC) below 30\%. In combination with immunohistochemistry and ddPCR, we confirmed the migration of hNSCs into the brain via nasal administration, which supported the efficacy of hNSCs in MPTP-treated mice, an animal model of Parkinson's disease. In conclusion, the present study is the first to report the application of ddPCR in the pharmacokinetics profile description of tracking of hNSCs in preclinical studies.

Acta Pharmacologica Sinica (2020) 41:572-580; https://doi.org/10.1038/s41401-019-0313-x

\section{INTRODUCTION}

Stem cells, especially neural stem cells (NSCs), are a valuable approach for regenerative medicine in Parkinson's (PD) disease, particularly for cell replacement therapy (CRT), both in the preclinical and clinical experimental settings [1,2]. It has now been recognized that stem cells can be delivered to remote injury sites to initiate damage, tissue repair, and tissue regeneration and stimulation. However, gaining insights into exogenous cell migration, tissue localization, and engrafting requires accurate tracking.

A variety of in vivo and ex vivo methods have been developed to identify transplanted cells $[3,4]$. Noninvasive in vivo technologies that evaluate the efficiency of transplantation and the migration of transplanted cells are commonly used for animal model studies and human clinical trials. Direct labeling and indirect labeling [3] are the two common strategies. For direct labeling, ${ }^{19}$ fluorine [5] and ${ }^{89}$ zirconium $\left({ }^{89} \mathrm{Zr}\right)$ [6] combined with singlephoton emission computed tomography (SPECT) and positron emission tomography (PET) imaging are the generally adopted techniques for their sensitivity $\left(\sim 10^{3}-10^{5}\right.$ cells). For the indirect bioluminescence imaging (BLI) labeling method, a comparative study of cell imaging with different nuclear reporter systems and substrates is performed using the appropriate radiolabeled probes for PET or SPECT imaging. Human T cells have been transfected with retroviral plasmids containing vectors encoding the human norepinephrine transporter (hNET), the human sodium iodide symporter, a human deoxycytidine kinase double mutant, and herpes simplex virus type 1 thymidine kinase reporter genes. The $\mathrm{hNET} /$ meta- $^{18} \mathrm{~F}$-fluorobenzylguanidine reporter system could detect less than $1 \times 10^{5} \mathrm{~T}$ cells, which is much more sensitive than the other BLI systems $\left(3 \times 10^{5}-1 \times 10^{6}\right.$ cells) [7].

Furthermore, magnetic resonance imaging (MRI), due to its inherent high spatial resolution, provides an alternative noninvasive method to study the fate of transplanted cells in vivo (allowing the detection of even $\sim 500$ labeled cells) [8]. Magnetic particle imaging (MPI)-based imaging systems represent a sensitive cell labeling and in vivo tracking method with a detection threshold as low as 200 cells [9-11], which allows exogenously labeled human stem cell tracking both in the murine brain and systemically [12]. The capabilities to image quantitatively, sensitively, and longitudinally makes MPI uniquely well suited for cell tracking applications to monitor the long-term localization and persistence of low cell populations, both for preclinical and clinic research [10]. In summary, the detection threshold of SPECT/PET, MRI, near-infrared live

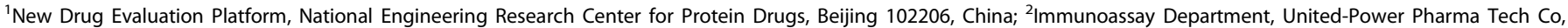

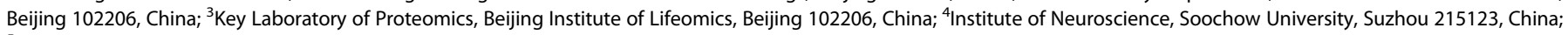

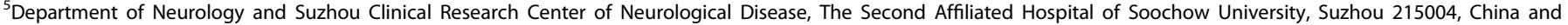
${ }^{6}$ Shanghai Angecon Biotechnology Co., Ltd, Shanghai 201318, China

Correspondence: Jie Fu (13311161618@163.com) or Hai-feng Song (songhf6811@163.com)

These authors contributed equally: Fang Wang, Zhe Wang

These authors jointly supervised this work: Jie Fu, Hai-feng Song

Received: 10 May 2019 Accepted: 30 September 2019

Published online: 8 November 2019 
imaging [13] and even the MPI-based imaging system is $\sim 2 \times 10^{2}-$ $2 \times 10^{5}$ cells, which might not be sufficient for some studies involving traces of cell tracking. In addition to the lack of sensitivity, considering the effects of labeled cells or transfected cells on cellular function, extensive study is needed to optimize efficient cell labeling or transfection without cell survival, proliferation, self-renewal, and multipotency $[14,15]$. Moreover, in vivo long-term images should be interpreted with caution due to the possibility of host cell internalization [16]. In contrast, ex vivo cell identification approaches, including immunohistochemical (IHC) and genetic techniques, could be complementary assays, which may be much more available and sensitive.

In this study, human neural stem cells (hNSCs) were administered nasally to treat a PD model in a preclinical study. Tracking a small number of cells in targeted sites (brain striatum) after nasal administration poses a very high technical challenge due to the sensitivity requirements. In the context of preclinical studies in xenogeneic transplantation, it is possible to use species-specific markers to distinguish donor cells from host cells. Here, under the premise that neither BLI nor SPECT can meet the sensitivity demand, a postmortem method based on species-specific markers amplified by droplet digital PCR (ddPCR) was performed. The ddPCR can be detected at the single molecule level to obtain an initial copy number or concentration of the target molecule, which has high sensitivity and accuracy. The distinctive feature of ddPCR is the separation of the reaction mixture into thousands to millions of partitions with the principle of Poisson distribution with the ability of absolute quantification of target nucleic acids [17]. Here, we determined the sensitivity using ddPCR to be $\sim 100$ cells and complementary to IHC. The validated ddPCR methodology provides a reference for the pharmacokinetic (PK) evaluation of preclinical cell therapeutics. We demonstrate that the function of CRT in PD relies on NSC regulation of the microenvironment instead of the direct difference and replacement of damaged cells.

\section{MATERIALS AND METHODS}

\section{Human NSCs}

The hNSCs and hNSCs stably transfected with hNET and the luciferase plasmid (hNET-Luc-NSCs) were provided by Shanghai Angecon Biotechnology Co., Ltd. (Shanghai, China).

\section{Animals}

C57BL/6 mice were obtained from Shanghai Lingchang Biotechnology Co., Ltd. (Shanghai, China) (Certificate No. 2013001817602).

The 1-methyl-4-phenyl-1,2,3,6-tetrahydropyridine (MPTP)-induced C57BL/6 PD mice model were prepared as previously reported [18] by Soochow University (Jiangsu, China). For BLI and ${ }^{89} \mathrm{Zr}$ imaging experiments, SD rats and C57BL/6 mice were used. For ddPCR and IHC experiments, MPTP C57BL/6 mice were used.

All animals were bred in a pathogen-free animal facility and provided ad libitum access to a standard diet (PMI LabDiet ; LabDiet, St Louis, MO, USA) and water. The illumination was $12 \mathrm{~h}$, alternating light and dark. At the beginning of the administration, the animals were 8 weeks old (Experimental animal license: SYXK (Su) 2015-0014).

The study was performed with the approval of the Ethical Committee of Soochow University. The animal study was strictly carried out in accordance with the recommendations in the Guide for the Care and Use of Laboratory Animals of the Beijing Institute of Lifeomics. Animal welfare and experimental procedures were carried out in accordance with the National Research Council's Guide for the use of laboratory animals.

\section{BLI experiments in vitro and in vivo}

We explored the sensitivity of the BLI method in vitro and in vivo (rats, mice) and the suitability for nasal administration of the study. In vitro, hNET-Luc-NSCs were seeded in 24 -well plates at $10^{3}, 10^{4}$, or $10^{5}$ in $0.5 \mathrm{~mL}$ of saline in replicate. Fluorescence scanning was performed after incubation with $5 \mu \mathrm{L}$ of fluorescein $(15 \mathrm{mg} / \mathrm{mL}$, PerkinElmer, USA) per well for $10-20 \mathrm{~min}$. For the in vivo experiments, different amounts of hNET-Luc-NSCs, $10^{6}$ (SD rats, $n=3$ ) $) ; 10^{5}$ (SD rats, $n=1$ \%); $5 \times 10^{4}$ (SD rats, $n=1$ ) $)$ or $5 \times 10^{5}$ (C57BL/6 mice, $\left.n=1 \%, 20^{*}\right)$ hNET-Luc-NSCs were intrastriatally administered.

Animals were anesthetized with a Matrx VIP 3000 anesthetic machine (Midmark Corporation, Dayton, $\mathrm{OH}$, USA) using 1\%-1.5\% isoflurane (RWD Life Science Biotechnology Co., Ltd., Shenzhen, China). Following anesthesia, $150 \mathrm{mg} / \mathrm{kg}$ luciferase substrate fluorescein (PerkinElmer, Wellesley, MA, USA) was administered via intraperitoneal injection and the animals were observed with an IVIS $^{\circ}$ Spectrum in vivo Bioluminescence and Fluorescent Imager (PerkinElmer). All animals were observed 20 min after intraperitoneal injection of the luciferase substrate fluorescein. Among them, two experimental animals, G1-F-02 and G2-F-02, were sacrificed after fluorescence scanning, and the brains were dissected. The brain tissue was then placed in a 12-well plate for in vitro fluorescence scanning. Intensity data from the in vivo and in vitro fluorescence scanning analyses were analyzed using GraphPad Prism Version 6 (GraphPad Prism Software Inc. San Diego, CA, USA).

${ }^{89} \mathrm{Zr}$-PET/CT in vitro and in vivo imaging experiments

${ }^{89} \mathrm{Zr}$-hNSCs were used in imaging experiments after the Radio-iTLC quality control and stability assay. First, in vitro sensitivity was studied by placing different amounts of ${ }^{89} \mathrm{Zr}$-hNSCs $\left(10^{2}, 10^{3}, 10^{4}\right.$, or $\left.10^{5}\right)$ into 96 -well plates $(0.1 \mathrm{~mL} /$ well with saline as a control) in duplicate. The 96-well plate was then static scanned for $10 \mathrm{~min}$ (scanning energy window: 350-650 keV) in a PET/CT SNPC-103 instrument (Pingsheng Medical Technology Co., Ltd.).

The in vivo detection sensitivity of ${ }^{89} \mathrm{Zr}$-hNSCs with PET/CT imaging was investigated via both striatal localization and nasal administration. SD rats were anesthetized with a Matrix VIP 3000 anesthetic machine (Midmark) using 1\%-1.5\% isoflurane (RWD Life Science Biotechnology Co., Ltd., Shenzhen, China). Animals were divided into G1-G4 groups, in which the G1-G3 groups $(n=$ 2 in each group) received striatal localization of different amounts $\left(10^{3}, 10^{4}\right.$, or $\left.10^{5}\right)$ of ${ }^{89} \mathrm{Zr}$-hNSCs using the brain stereotactic instrument 68001 (RWD Life Science) combined with a microinjector $(5 \mu \mathrm{L})$ (Gaogee Industry \& Trade Co., Ltd. Shanghai, China). A PET/CT brain static scan (scan energy window: 350-650 keV) was performed for $10 \mathrm{~min}$ at $4 \mathrm{~h}$ postadministration. In the $\mathrm{G} 4$ group $(n=3)$, rats were nasally administered $2 \times 10^{6}{ }^{89} \mathrm{Zr}$-hNSCs. The PET/CT brain static scan of the small animals was acquired for $10 \mathrm{~min}$ at different time points $(10 \mathrm{~min}, 35 \mathrm{~min}, 60 \mathrm{~min}, 4 \mathrm{~h}, 24 \mathrm{~h}$, $72 \mathrm{~h}$, and $168 \mathrm{~h}$ ). Upon the end of the 168-h observation period, the animal was sacrificed, and the whole brain was obtained and subjected to a PET/CT static scan for $10 \mathrm{~min}$ (scanning energy window: $350-650 \mathrm{keV}$ ).

The image reconstruction was performed followed by data acquisition completion. The reconstruction algorithm was OSEM $3 D+$ PSF (the number of iterations was 5 ). The radioactive uptake values of the nasal cavity and brain regions of interest of each animal were analyzed using PMOD software (PMOD Technologies LTD, Zurich, Switzerland). The \%ID/g of the region of interest was calculated using the following formula:

$$
\begin{gathered}
\% \mathrm{ID} / \mathrm{g}=[\text { radioactive substance uptake per unit volume of } \\
\text { interest }(\mu \mathrm{Ci} / \mathrm{g}) / \text { total injected dose }(\mu \mathrm{Ci})] \times 100 .
\end{gathered}
$$

Data were analyzed using Excel 2007 (Microsoft Corporation, Redmond, WA, USA) and Prism software 6.0.

\section{Histology}

MPTP mice nasally administered $2 \times 10^{6}$ hNSCs were sacrificed, and blood samples were collected from the heart apex into an 
anticoagulation tube. The heart was then fully perfused with the saline followed by $\sim 30 \mathrm{~mL}$ of precooled $4 \%$ paraformaldehyde. Dissections were performed immediately upon paraformaldehyde fixation. The brain and the spinal cord were placed in a $50 \mathrm{~mL}$ centrifuge tube containing 4\% paraformaldehyde at 10 times the volume of the tissue. Peripheral tissues [heart, liver, spleen, lung, kidney, testis and epididymis (male), and ovary (female)] were placed in $10 \%$ formalin for paraffin embedding and sliced (thickness: $20 \mu \mathrm{m}$ ) with a paraffin slicer (Leica Biosystems Inc. Buffalo Grove, USA).

\section{Immunofluorescence}

After fixing for $4 \mathrm{~h}$ with $4 \%$ paraformaldehyde, the tissues were dehydrated with a gradient of $15 \%$ and $30 \%$ sucrose solution, respectively. According to the position coordinates of the various parts (striatum, substantia nigra, cortex, hippocampus, cerebellum) in the mouse brain map, a frozen slicer was used to serially slice the brain tissues (thickness, $20 \mu \mathrm{m}$ ) on a cryostat (Leica, Wetzlar, Germany). Brain slices were washed three times with PBS for 5 min each time. Then, the slices were blocked with PBS containing $0.1 \%$ Triton X-100 and 2\% BSA for $1 \mathrm{~h}$. Then, the mouse STEM 121 antibody (1:500; Takara Bio Inc., Japan) and SOX2 (1:500, Merck Millipore, Germany) were incubated with the slices at $4{ }^{\circ} \mathrm{C}$ overnight, followed by three washes with PBS for $5 \mathrm{~min}$ each time, followed by incubation with 488 anti-mouse secondary antibody (1:1000, Invitrogen) and 555 anti-rabbit secondary antibody (1:1000, Invitrogen) for $1 \mathrm{~h}$ at room temperature. After three washes with PBS for 5 min each time, the slices were dried and sealed with VECTASHIELD ${ }^{\infty}$ Mounting Medium with DAPI from Vector Laboratories Inc. (CA, USA). The cells were photographed under a Zeiss fluorescence microscope (Axio ScopeA1, Goettingen, Germany).

\section{ddPCR tested samples in vivo}

The MPTP-induced mouse PD model group was used for the in vivo efficacy study that accompanied the PK study. The hNSCs $\left(2 \times 10^{6}\right.$ cells in $8 \mu \mathrm{L}$ of $\mathrm{NaCl}$ per mouse) or control $\mathrm{NaCl}$ ( $8 \mu \mathrm{L} /$ mouse) were administered via a single intranasal administration. The frozen and paraffin sectioned samples from this study were harvested from three dose groups (Groups 1-3), and the blood samples were from Group 1 and Group 3. Detailed information about the time points of collection and the number of slices is shown in Table 1.

\section{Sample harvest and management}

A total of 333 frozen mouse brain slices (striatum, olfactory bulb, hippocampus, substantia nigra, cerebellum, spinal cord), 342 mouse paraffin slices (heart, liver, spleen, lung, kidney, genitals) and 20 whole blood samples were employed in the ddPCR study. All samples were produced by the Institute of Neuroscience, Soochow University, and sample analysis was performed at United-Power Pharma Tech Co. Ltd., Beijing. According to this methodology, four slices (the thickness of each slice was $20 \mu \mathrm{m}$ ) of frozen brains or paraffin brains were used for genomic DNA (gDNA) extraction.

\section{Sample preparation and ddPCR}

gDNA was extracted from frozen and paraffin tissues (four slices per reaction) using a QIAamp DNA FFPE tissue kit (Qiagen, Crawley, UK) according to the manufacturers' protocols. Whole blood gDNA was extracted using the DNeasy Blood \& Tissue Kit (QIAGEN). The gDNA concentration was determined using Thermo Scientific ${ }^{\mathrm{TM}}$ NanoDrop ${ }^{\mathrm{TM}}$ One (Thermo Scientific Inc., Waltham, USA) and kept at -60 to $-90^{\circ} \mathrm{C}$ for further ddPCR analysis.

The endogenous reference $\beta 2$-microglobulin $(\beta 2 \mathrm{M})$ gene was used as a species-specific marker because of its excellent primer and probe performance in the $\mathrm{ddPCR}$ system. The primers and probes were synthesized by Sangon Biotech Co., Ltd. (Shanghai,
China). Forward primer (5'-3'): GCAAGGACTGGTCTTCTATC; reverse primer $\left(5^{\prime}-3^{\prime}\right)$ : ATACACAACTTTCAGCAGCT; probe $\left(5^{\prime}-3^{\prime}\right)$ : FAM-CCCCCACTGAAAAAGATGAGTATGCC-BHQ1.

Each ddPCR analysis batch contained a set of quality control samples (LQC, $n=2$ ), negative control (NTC, $n=2$ ) samples, and blank (Blank, $n=2$ ) samples. NTC sample: $d_{d P C R}{ }^{\text {TM }}$ Supermix for Probes (No dUTP) (Bio-Rad), probes, primers, and gDNA extracted from blank tissues as a template. Blank sample: dyes, probes, primers, and diethyl pyrocarbonate (DEPC)-treated water as a template.

ddPCR was performed in a QX200 ${ }^{\mathrm{TM}}$ AutoDG $^{\mathrm{TM}}$ Droplet Digital ${ }^{\mathrm{TM}}$ PCR System (Bio-Rad, Hercules, CA, USA). First, QPCR mix was prepared $\left(20 \mu \mathrm{L} /\right.$ reaction) with $10 \mu \mathrm{L}$ of ddPCR $^{\text {TM }}$ Supermix for Probes, $1.6 \mu \mathrm{L}$ of forward primer $(10 \mu \mathrm{M}), 1.6 \mu \mathrm{L}$ of reverse primer $(10 \mu \mathrm{M}), 0.5 \mu \mathrm{L}$ of TaqMan Probe $(10 \mu \mathrm{M})$, and gDNA template $(6.3 \mu \mathrm{L}, 100-500 \mathrm{ng})$. The microplates were transferred to the Automated Droplet Generator (Bio-Rad) to generate droplets. Further, PCR was performed according to the amplification conditions: $5 \mathrm{~min}$ at $95^{\circ} \mathrm{C} ; 40$ cycles of $30 \mathrm{~s}$ at $95^{\circ} \mathrm{C}, 40 \mathrm{~s}$ at $60^{\circ} \mathrm{C}$; and finally $10 \mathrm{~min}$ at $98^{\circ} \mathrm{C}$ and storage at $12^{\circ} \mathrm{C}$. Finally, the amplified products were transferred to the $\mathrm{QX200^{ \textrm {TM } }}$ Droplet Reader for analysis.

\section{ddPCR methodology validation}

Quality control samples were prepared in three types of tissues, including frozen slices, paraffin slices and whole blood, for the method validation study. Blank tissue matrix was used as a negative control (NTC). The 500 hNSCs were spiked into four frozen mouse cerebellum ( 2 batches)/striatum (1 batch) tissue slices (or four mouse peripheral (liver) tissue paraffin slices, or $200 \mu \mathrm{L}$ whole blood) and genomic DNAs were extracted as the lower quality control (LQC) and lower limit of quantitation (LLOQ). The above gDNA samples were kept at -60 to $-90^{\circ} \mathrm{C}$. Two analysts completed three analysis batches in two days. The interbatch and intra-assay precision of the method is expressed as a percent of the coefficient of variation $(\% \mathrm{CV}=[$ (standard deviation/mean) $\times 100]$ ).

Long-term and freeze-thaw stabilities were investigated. In the long-term stability study, the samples above the LQC (500 cells/4 tissue slices, or $200 \mu \mathrm{L}$ whole blood) were stored at -60 to $-90^{\circ} \mathrm{C}$ for 1, 2, and 3 months. Four LQC replicates were used for evaluation at each time point. In the freeze-thaw stability study, the above LQCs were stored at -60 to $-90{ }^{\circ} \mathrm{C}$ for one freeze-thaw cycle and two cycles with four LQC replicates at each time point. The acceptable standards for stability samples are as follows: duplicate well CV\%: $\leq 30 \%$, and $2 / 3$ of the QCs met the acceptance criteria; the AR\% was based on a baseline lower than $20 \%$.

\section{ddPCR data analysis and acceptance criteria}

Each sample was run in duplicate during the $\mathrm{ddPCR}$ detection process. The ddPCR data are shown as copies $/ 20 \mu \mathrm{L}$ and were reported by the QX200 ${ }^{\mathrm{TM}}$ Droplet Reader control software QuantaSoft $^{\mathrm{TM}}$ 1.7.4 (Bio-Rad Inc.), and the data were analyzed using Excel 2013 (Microsoft Inc., Office 2013).

The samples with more than 10000 droplets could be defined as effective data; otherwise, the sample could be reanalyzed. Each analysis batch contained one LQC $(n=4)$, and at least $2 / 3$ of the QC samples must satisfy the acceptance criteria; the \%CV was lower than $30 \%$.

The threshold line was defined according to the positive and negative droplet response values of the NTC and blank per analytical batch.

The cutoff value of the positive droplet per analysis batch was calculated using the Excel determination of the LOD (Fig. 1a). The cutoff value of each analytical batch must be lower than 12. A calculated cutoff value of 12 indicates the potential presence of sample contamination, which indicates unacceptance of the analytical batch. 
Table 1. ddPCR samples information

\begin{tabular}{|c|c|c|c|}
\hline MPTP + hNSC (Group1) & $\begin{array}{l}4 \mathrm{~h}(n=6), 24 \mathrm{~h}(n=6), 3 \text { days }(n=6) \\
7 \text { days }(n=6), 14 \text { days }(n=6), 28 \text { days } \\
(n=5), 56 \text { days }(n=3), 90 \text { days }(n=3)\end{array}$ & $\begin{array}{l}4 \mathrm{~h}(n=6), 24 \mathrm{~h}(n=6), 3 \text { days }(n=6), \\
7 \text { days }(n=6), 14 \text { days }(n=6), 28 \text { days } \\
(n=5), 56 \text { days }(n=3), 90 \text { days }(n=3)\end{array}$ & $4 \mathrm{~h}(n=5), 24 \mathrm{~h}(n=5), 3$ days $(n=5)$ \\
\hline Normal + hNSC (Group 2) & $24 \mathrm{~h}(n=6), 7$ days $(n=6)$ & $4 \mathrm{~h}(n=6), 7$ days $(n=6)$ & / \\
\hline MPTP + NaCl (Group 3) & $4 \mathrm{~h}(n=3), 7$ days $(n=3)$ & $4 \mathrm{~h}(n=3), 7$ days $(n=3)$ & $4 \mathrm{~h}(n=5), 24 \mathrm{~h}(n=5), 3$ days $(n=5)$ \\
\hline
\end{tabular}

a

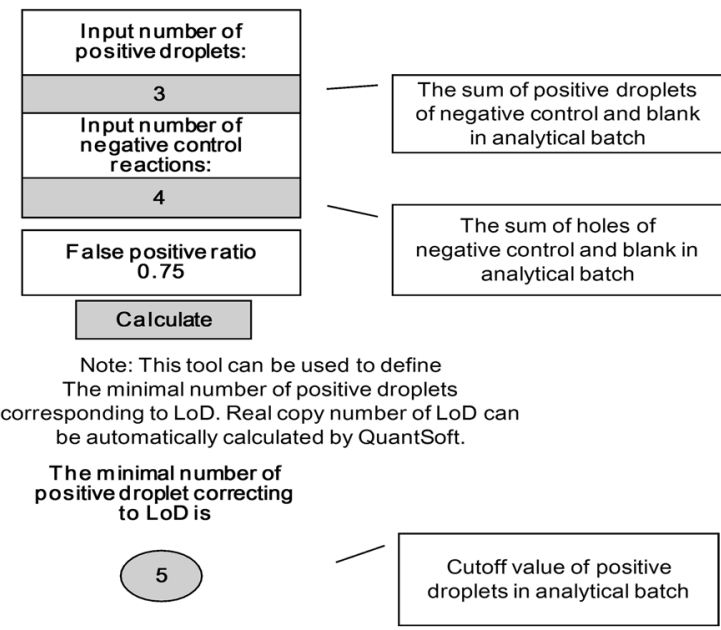

b

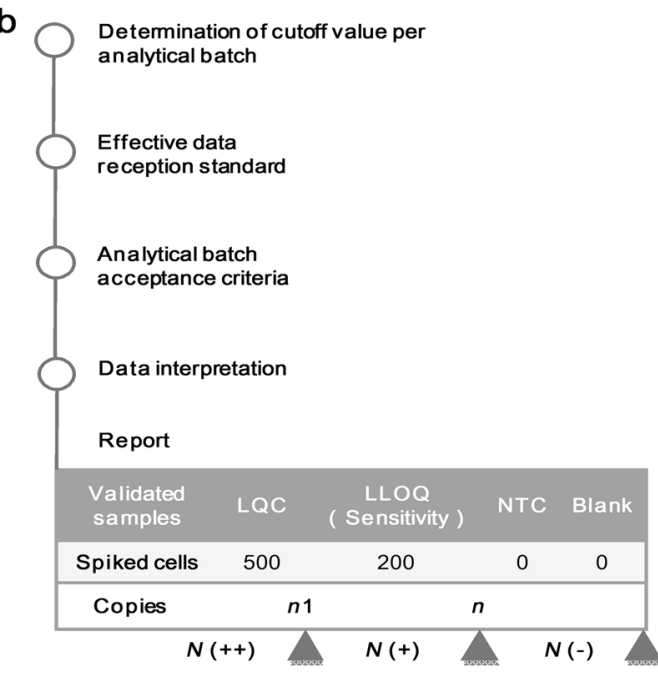

Fig. 1 Data explanation of ddPCR. a Cutoff value of the positive droplet per analytical batch. $\mathbf{b}$ ddPCR data report process

Data interpretation process

To compare the results of immunofluorescence/IHC, we defined the results of ddPCR by $-/+/++$, and the standard of definition can be found in the following data interpretation process (Fig. 1b).

Assuming that the cutoff value of the software calculation analysis batch is $n$, the number of positive droplets of the sample to be tested is $N$, and the number of positive droplets of the LLOQ is $n 1$.

If $N \geq \mathrm{n} 1$, the sample is defined as ++ (strongly positive);

if $\mathrm{n} 1>N \geq n$, the sample is defined as + (positive); and

if $n>N$, the sample is defined as - (negative).

Statistical analysis

Differences between the grafted cells at different dosing groups after transplantation were evaluated using GraphPad Prism Version 6 (GraphPad Prism Software Inc. San Diego, CA, USA). Data are expressed as the means $\pm S E M$, and differences are considered significant at $P<0.05$.

\section{RESULTS}

$B L I$ in vitro sensitivity

hNSCs at different cell numbers were added to a 24-well plate for fluorescence imaging. The mean fluorescence intensity (MFI) derived from the blank control well, $1 \times 10^{3}$ cells/well, $1 \times 10^{4}$ cells/well was $9.90 \times 10^{4} \mathrm{p} / \mathrm{s}, 9.72 \times 10^{4} \mathrm{p} / \mathrm{s}(P=0.928$ vs control), and $1.35 \times 10^{5} \mathrm{p} / \mathrm{s}(P=0.212$ vs control), respectively. The MFI at $1 \times 10^{5}$ cells per well (mean $\left.=6.59 \times 10^{5} \mathrm{p} / \mathrm{s}\right)$ was significantly higher than that of the blank control well $(P=0.001)$. For the BLI, the in vitro sensitivity is $1 \times 10^{5}$ cells (Fig. 2 a).
$B L I$ in vivo experiments

The animals (SD rats and C57BL/6 mice) were subjected to BLI after striatum and nasal administration of hNSCs, and the interested areas (brain nearby nose) were scanned for fluorescence intensity, and the fluorescence intensity of the animal back was the control (the area was the same as the target area) (Supplementary Fig. 1).

Different amounts of hNSCs were injected into the striatum of SD rats at doses of $1 \times 10^{6}, 1 \times 10^{5}$, and $5 \times 10^{4}$. Fluorescence signals were captured 20 min after administration. At the site of administration, $10^{6}$ cell injections incurred an average fluorescence intensity of $1.27 \times 10^{6} \mathrm{p} / \mathrm{s}$ compared with the control of $2.80 \times 10^{5} \mathrm{p} / \mathrm{s}(P=0.021)$ (Fig. 2b). After $2 \mathrm{~h}$, the signals were not significantly different between the site of administration and the control site. Moreover, injection of $5 \times 10^{4}$ and $1 \times 10^{5}$ hNSCs yielded no detectable signals compared to controls. In addition, nasal administration of $10^{6} \mathrm{hNSCs}$ yielded a fluorescent intensity of $5.47 \times 10^{5} \mathrm{p} / \mathrm{s}$ at $24 \mathrm{~h}$, which was indistinguishable from the control $\left(3.44 \times 10^{5} \mathrm{p} / \mathrm{s}\right)$.

The in vivo BLI sensitivity of C57BL/6 mice was found to be similar to that in rats for the mice administered $5 \times 10^{5}$ hNSCs $20 \mathrm{~min}$ after intracerebral striatum injection. The total fluorescence was observed at the site of administration (i.e., brain) $(1.69 \times$ $\left.10^{6} \mathrm{p} / \mathrm{s}\right)$ and the back control $\left(1.10 \times 10^{5} \mathrm{p} / \mathrm{s}\right) \quad(P<0.01)$. No significant signals were detected in the other groups or at the other time points.

To verify whether the $B L I$ in vivo sensitivity was hindered by the depth of the targeted location, we dissected the brain tissue in G1F-02 $\left(1 \times 10^{6}\right.$ cells into the striatum $)$ and G2-F-02 $\left(1 \times 10^{6}\right.$ cells in nasal cavity). The fluorescent intensities were $2.36 \times 10^{5} \mathrm{p} / \mathrm{s}$ and $9.41 \times 10^{4} \mathrm{p} / \mathrm{s}$, respectively, in comparison to the control $(1.01 \times$ 


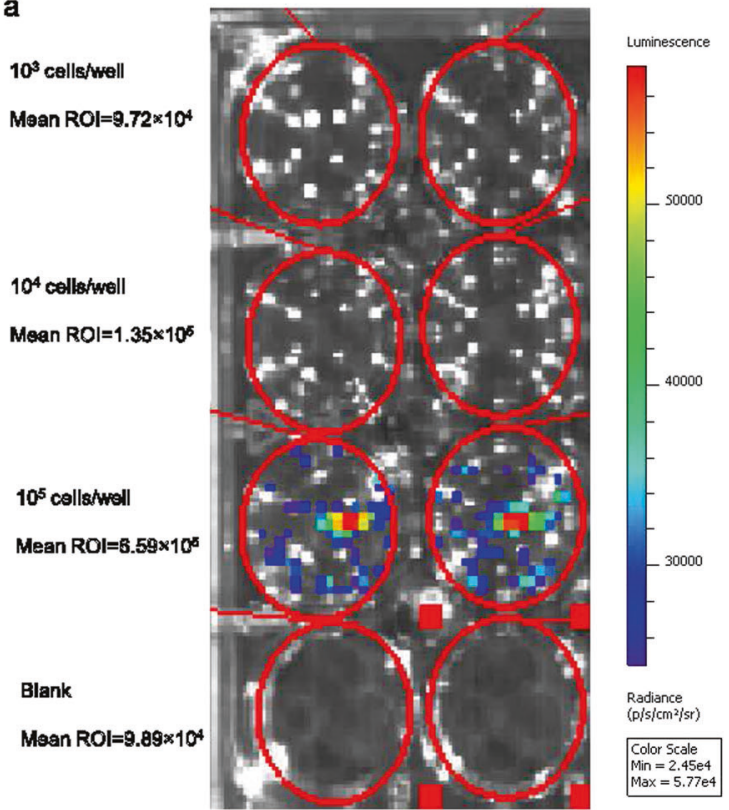

b

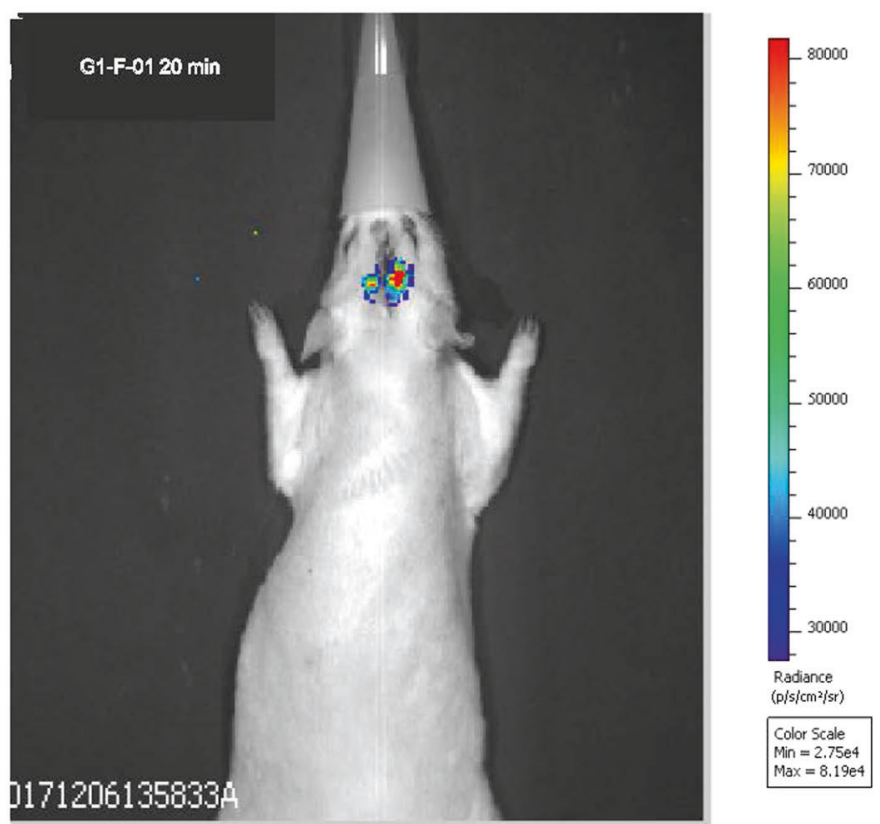

Fig. 2 Typical imaging of in vitro and in vivo intrastriatal administration. a Different cell numbers $\left(1 \times 10^{3}-1 \times 10^{5}\right)$ of hNET-Luc-hNSCs were added to a 24 -well plate for fluorescence imaging. The in vitro detection threshold was $\sim 10^{5}$ cells. b Typical imaging of in vivo intrastriatal administration $\left(10^{6}\right.$ hNET-Luc-hNSCs). The in vivo detection threshold was $\sim 10^{6}$ cells

$10^{5} \mathrm{p} / \mathrm{s}$ ). Taken together, although the cells were successfully administered into the brain areas of interest, the tracing of these cells was not successful due to insufficient sensitivity of the fluorescent imaging.

${ }^{89} \mathrm{Zr}$ in vitro PET/CT imaging experiments

The results of an in vitro PET/CT scan of 96-well plates showed that with an increase in ${ }^{89} \mathrm{Zr}$-hNSCs, the total radioactivity per well increased. Among them, the dose of ${ }^{89} \mathrm{Zr}$-hNSCs of $10^{2}$ cells was relatively low, the interwell CVs was $108 \%$, and the CVs of doses of $10^{3}, 10^{4}, 10^{5}$ cells/well were $<10 \%$, indicating accurate quantification. The sensitivity of the ${ }^{89} \mathrm{Zr}$ in vitro PET/CT imaging was $10^{3}$ cells (Fig. 3a).

In the G1-G3 groups, different amounts of ${ }^{89} \mathrm{Zr}$-hNSCs were stereotactically localized into the normal SD rats, and a PET/CT brain scan was performed $4 \mathrm{~h}$ after the striatum injection. The results showed that radioactive signals were detected in the right brains of the G1-G3 groups. In addition, with the increase in ${ }^{89} \mathrm{Zr}$ hNSC administration, the signals were enhanced in the whole brain. The distribution profile of ${ }^{89} \mathrm{Zr}$-hNSCs was different between the G1 and G2 groups. ${ }^{89} \mathrm{Zr}$-hNSCs were mainly distributed in the striatum of the $\mathrm{G} 1$ group ( $10^{3}$ cells). Radioactive materials were mainly distributed in the striatum and cortex in the G2 group $\left(10^{4}\right.$ cells). ${ }^{89} \mathrm{Zr}$-hNSCs were mainly distributed in the striatum, cortex, and hippocampus in the $\mathrm{G} 3$ group $\left(10^{5}\right.$ cells) (Fig. $\left.3 \mathrm{~b}, \mathrm{c}\right)$.

In the G4 group, ${ }^{89} \mathrm{Zr}$-hNSCs were intranasally administered $2 \times$ $10^{6}$ cells per rat. The PET/CT brain scans were performed at different time points. The results showed that the radioactive material was mainly distributed in two locations: in the nasal olfactory area and the ethmoid position (Fig. 4). Some of the cells may enter the esophagus through the respiratory area. The radioactive uptake values of the nasal cavity and the olfactory bulb gradually decreased over time, and the radioactive uptake values of the pituitary and pons first increased and then decreased. The radioactive uptake values of the other brain regions, such as the frontal lobe, parietal lobe, striatum, hippocampus, and VTM, were relatively low, and no signals were observed after $4 \mathrm{~h}$. In summary, when ${ }^{89} \mathrm{Zr}$-hNSCs were administered via the nasal cavity to SD rats, ${ }^{89} \mathrm{Zr}$-hNSCs were predominantly distributed in the nasal cavity, and a limited amount of signal was captured in the brain (below the detection limit). In addition, when we performed nasal administration, the ${ }^{89} \mathrm{Zr}$-hNSCs may be excreted and metabolized after entering the esophagus through the respiratory tract (occurrence rate was 1/3). Taken together, no radioactive signals were detected $4 \mathrm{~h}$ after nasal administration in vivo. The rats were sacrificed, and the brains were harvested and scanned with PEC/CT at $168 \mathrm{~h}$. No signals were detected ex vivo, confirming that the radioactive substance did not enter the brain (or the level was below the detection limit).

\section{The ddPCR method validation}

A total of eight pairs of primers and TaqMan probe candidates involving two kinds of species-specific genes were compared and optimized according to ddPCR performance [19 20]. Different amounts $\left(0,10^{2}, 10^{3}, 10^{4}, 10^{5}, 10^{6}\right)$ of hNSCs were spiked into $25 \mathrm{mg}$ of brain or four tissue slices, and the total gDNA was extracted. The optimized set of primers and probe were used to establish standard curves (Supplementary Fig. 2). The mean values (copies/20 $\mu \mathrm{L}$ ) of triplicate measurements of the experimental samples were $6.7(\% \mathrm{CV}=59.7 \%), 24.7 \quad(\% \mathrm{CV}=29.8 \%), 52.7$ $(\% \mathrm{CV}=13.3 \%), 752.7(\% \mathrm{CV}=4.8 \%)$, and $4860.0(\% \mathrm{CV}=3.8 \%)$, respectively. The sensitivity (lower limit of quantification, LLOQ) was $100-200$ cells/4 slice tissues. The LQC was prepared using 500 cells spiked in four slice tissues. Method validation was run as the LQC indicated in three types of biological matrices: frozen slices, paraffin slices, and whole blood.

The precision, selectivity, and stability (including two cycles of freeze-thaw stability, 3-month long-term stability) samples met the method validation requirements of biological samples [21-24]. The method sensitivity values ( $n 1)$ were $5.3,13$, and 4.8 for the frozen section, paraffin section, and whole blood, respectively. Sample bioanalysis involved a total of 33 ddPCR analyses. For the intra-assay precision, the CVs of the LQC ranged from $2.8 \%$ to $26.2 \%, 6.1 \%$ to $24.5 \%$, and $23.4 \%$ to $28.4 \%$ in the frozen section, paraffin section, and whole blood, respectively. For the interassay precision, the CVs of the LQC were $23.7 \%, 21.3 \%$, and $25.4 \%$ in the three matrices. Taken together, the intra- and interassay precision 
a

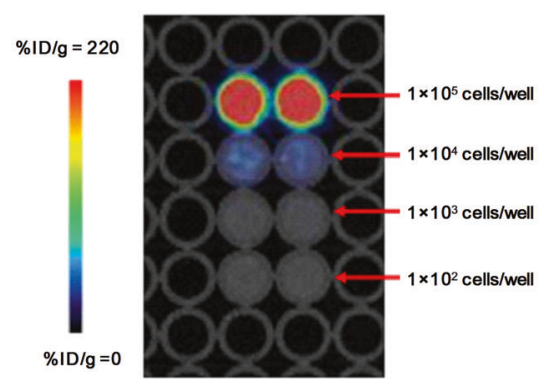

C

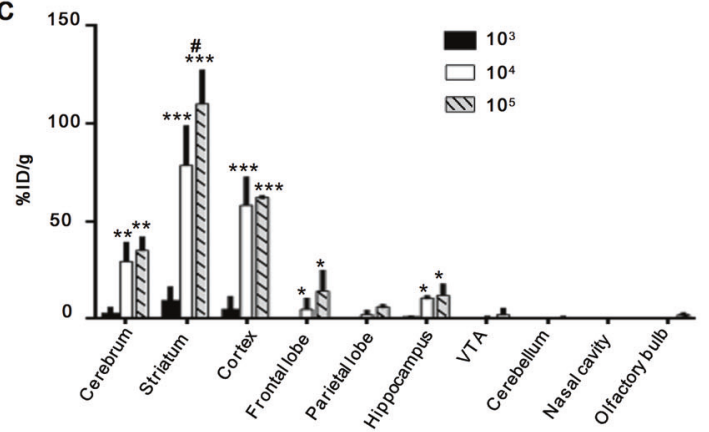

b

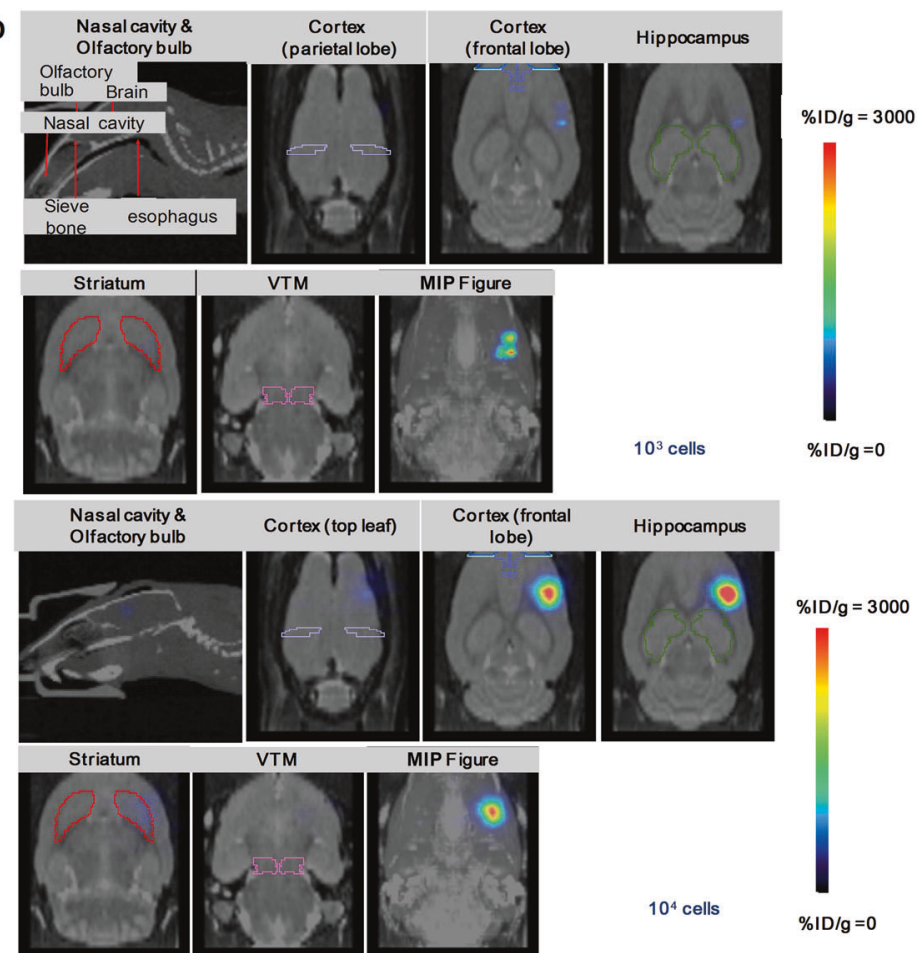

Fig. 3 Biodistribution of ${ }^{89} \mathrm{Zr}$-hNSCs at $4 \mathrm{~h}$ after intrastriatal administration. a Different cell numbers $\left(1 \times 10^{2}-1 \times 10^{5}\right)$ of ${ }^{89} \mathrm{Zr}$-hNSCs were added to a 96 -well plate for PET/CT imaging. The detection threshold was $\sim 10^{3}$ cells. $\mathbf{b}$ Typical brain imaging of the biodistribution of ${ }^{89} \mathrm{Zr}$ hNSCs at $4 \mathrm{~h}$ after intrastriatal administration of $10^{3}$ and $10^{4}{ }^{89} \mathrm{Zr}-\mathrm{hNSCs}$ (individual G1-F-01). c \% $\%$ ID/g representing the ${ }^{89} \mathrm{Zr}$-hNSC biodistribution in the region of interest of the brain after intrastriatal administration of $10^{3}, 10^{4}$, and $10^{5}{ }^{89} \mathrm{Zr}$-hNSCs. ${ }^{*} P<0.05$, ${ }^{* *} P<0.01$, ${ }^{* * *} P<0.001$ vs $10^{3}$ intrastriatal administration group. ${ }^{\#} P<0.05$ vs. $10^{4}$ intrastriatal administration group. VTM, midbrain ventral tegmental area
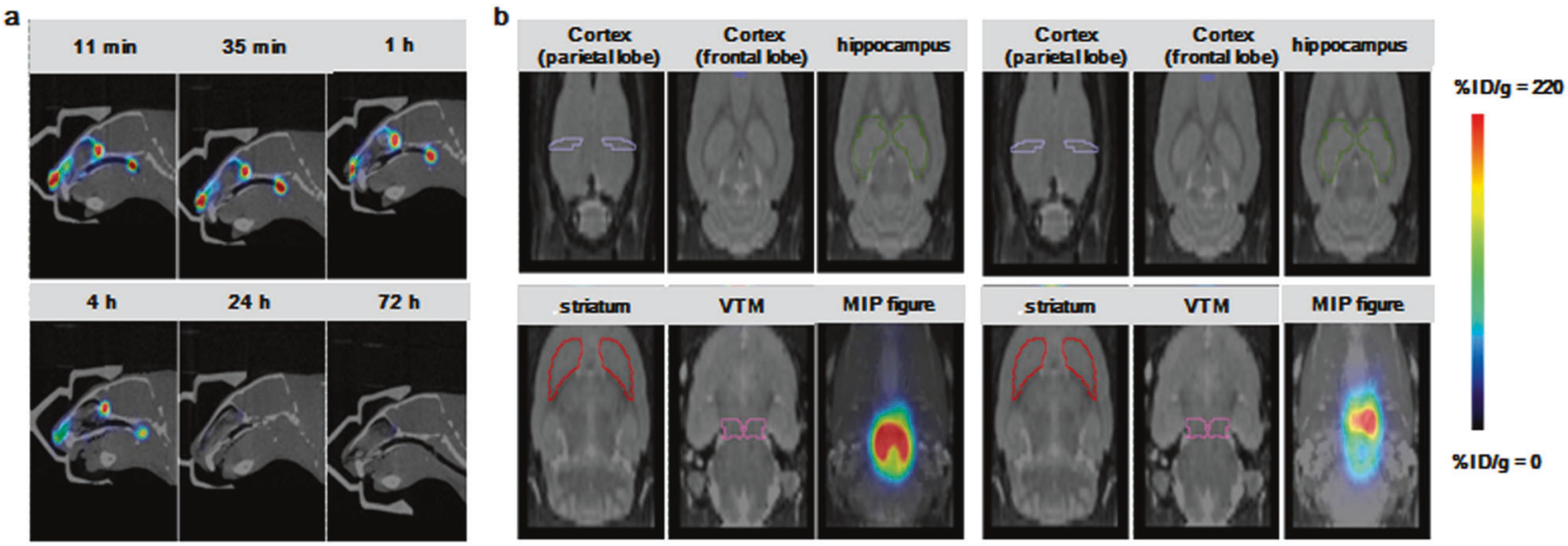

Fig. 4 Biodistribution of ${ }^{89} \mathrm{Zr}$-hNSCs postnasal administration. a Typical images of ${ }^{89} \mathrm{Zr}$-hNSCs in the nasal cavity and olfactory bulb at 11 min, $35 \mathrm{~min}, 1 \mathrm{~h}, 4 \mathrm{~h}, 24 \mathrm{~h}$, and $72 \mathrm{~h}$ after nasal administration of $2 \times 10^{6}{ }^{89} \mathrm{Zr}$-hNSCs per rat (individual G4-F-02). b Cortex (parietal lobe), cortex (frontal lobe), hippocampus, striatum, and midbrain ventral tegmental area (VTM) scanning using PET/CT and whole brain MPI imaging at $35 \mathrm{~min}$ (left) and $4 \mathrm{~h}$ (right) postnasal administration of $2 \times 10^{6}{ }^{89} \mathrm{Zr}$-hNSCs per rat (individual G4-F-01)

$\% \mathrm{CV}$ values were lower than $30 \%$ throughout the bioanalytical process.

The long-term stability within 3 months of the three matrices met acceptable standards. The freeze-thaw stability results showed that in frozen slices and the whole blood matrix, the $\% C V$ results of one and two freeze-thaw cycles of the LQC met the acceptable standard. In the paraffin section, one and two freezethaw cycles of the $\% C V$ were $14.1 \%$ and $41.8 \%$, respectively. The baseline value of one freeze-thaw sample was $65.6 \%$ compared with the baseline \%AR for two freeze-thaw samples, which failed to meet the acceptance criteria. Hence, the results indicated that the paraffin slices must be analyzed after no more than one freeze-thaw cycle.

Double-blind detection of hNSCs in animal models using immunofluorescence detection and ddPCR

Based on the biodistribution profile using $\operatorname{ddPCR}$, we found that although ddPCR can accurately quantify the distribution of hNSCs with a sensitivity of 100-200 cells/4 brain frozen slices, the Poisson distribution has a greater influence on the positive detection rate because of the trace distribution of hNSCs in animal models. To compare the advantages and disadvantages of the 


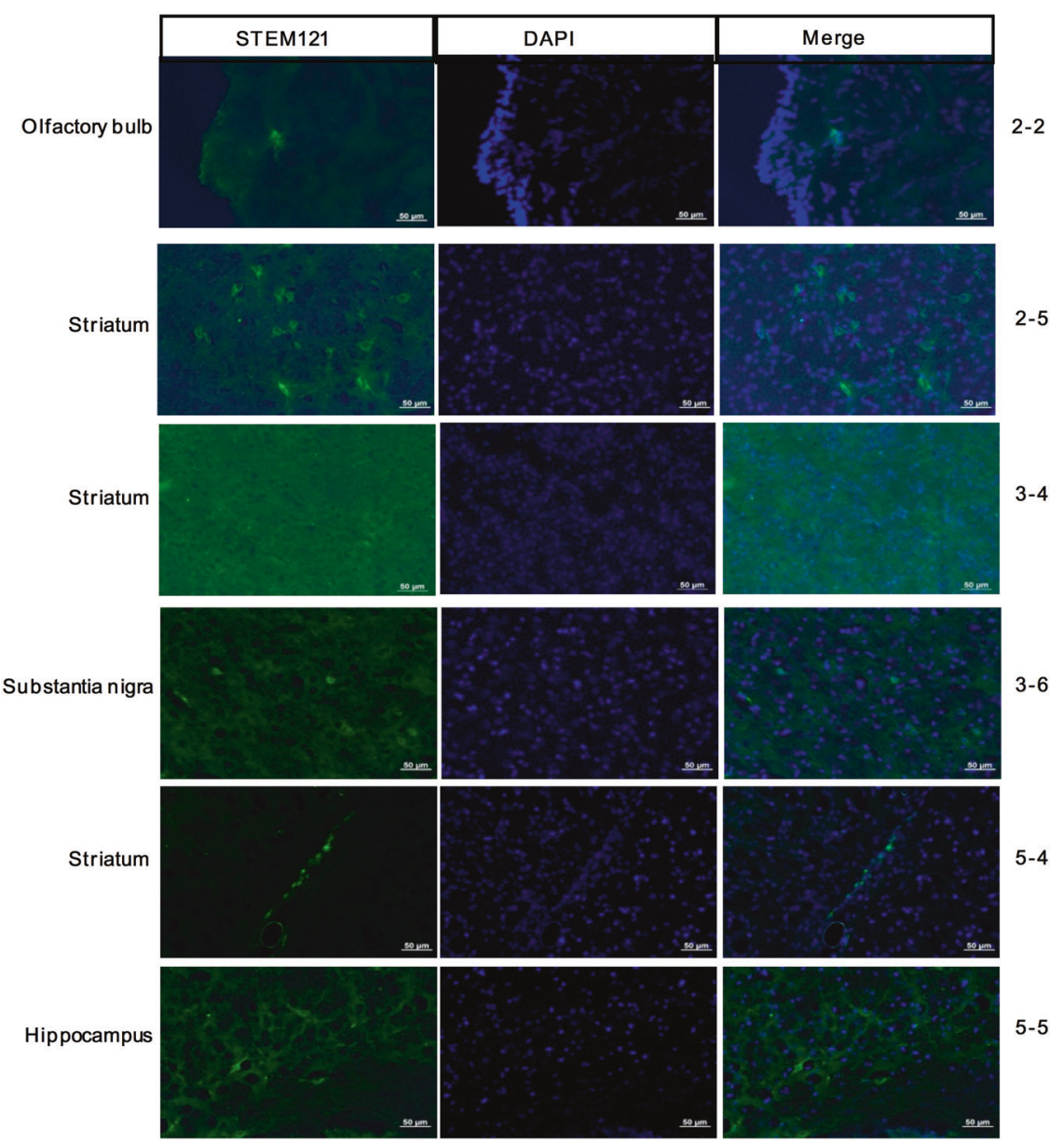

Fig. 5 Typical immunofluorescence images of hNSCs postnasal administration. The rats were nasally administered $2 \times 10^{6} \mathrm{hNSCs}$, and the brain (striatum, cortex, and hippocampus regions) immunofluorescence was determined at $24 \mathrm{~h}, 3$ days, 4 days, and 7 days (STEM121, 1:500). Only $24 \mathrm{~h}$ immunofluorescence images were shown

immunofluorescence and ddPCR methods for cell distribution, a double-blind method was prepared. The results indicated a high coincidence rate between the two methods, although differences did exist between duplicate wells due to the Poisson distribution of the positive droplets (Fig. 5 and Table 2).

hNSC biodistribution using ddPCR in MPTP model mice

After MPTP model mice were administered $10^{6} \mathrm{hNSCs}$ nasally, ddPCR analysis of the frozen brain slices demonstrated that $+/++$ signals were detected, representing that hNSCs could be detected in the striatum at $24 \mathrm{~h}, 3$ days, 7 days, 14 days, and 90 days (positive individuals $=1-2$ ). In addition, $+/++$ signals were detected in the hippocampus at 3, 7, 14, and 28 days (positive individuals $=1-2$ ), and $+/++$ signals were detected in the cerebellum at $4 \mathrm{~h}, 24 \mathrm{~h}, 3$ days, 14 days, and 56 days (positive individuals =1-2). In the spinal cord, + signals were detected in one individual at $4 \mathrm{~h}$, and no positive signals were detected in the olfactory bulb or the substantia nigra. In contrast, $+/++$ signals were detected in the striatum (positive individual =1), substantia nigra (positive individual $=1$ ), and cerebellar stroma (positive individuals $=1-3$ ) at $24 \mathrm{~h}$ and 3 days after intranasal administration in the normal group (Fig. 6).

After MPTP model mice were administered hNSCs nasally, ddPCR analysis of peripheral paraffin tissues showed that ++ signals were detected in the heart at $24 \mathrm{~h}$ and 14 days (positive individual $=1$ ), and $+/++$ signals were detected in the liver at 3 , 14,28 , and 56 days (positive individuals $=1,1,2,1$, respectively). Moreover, $+/++$ signals were detected in the spleen at 14,28 ,
Table 2. Double-blind detection of hNSCs in animal models using Immunofluorescence and ddPCR

\begin{tabular}{lllll}
\hline Sample & Immunofluorescence & ddPCR & Copies/20 $\mu \mathrm{L}^{\mathrm{a}}$ & Copies $/ 20 \mu \mathrm{L}^{\mathrm{b}}$ \\
\hline $2-2$ (2) & + & $+/-$ & 8 & 2 \\
$2-4$ (2) & - & - & 0 & 0 \\
$2-5$ (2) & + & $+/-$ & 0 & 8 \\
$3-4$ (2) & - & - & 0 & 0 \\
$3-6$ (2) & + & + & 4.6 & 17.8 \\
$5-4$ (2) & + & + & 16 & 12.6 \\
$5-5$ (2) & + & + & 0 & 32 \\
$5-6$ (2) & + & + & 0 & 50 \\
\hline${ }^{\mathrm{a} C}$ Cutoff value is 5 for the batch & \\
${ }^{\mathrm{b} C u t o f f}$ value is 9 for the batch
\end{tabular}

and 56 days (positive individuals $=1,4,3$, respectively), + signals were detected in the lungs at 14 and 28 days (positive individuals $=3,3$, respectively), and $+/++$ signals were detected in the kidneys at $14,28,56$, and 90 days (positive individuals $=2$, $3,2,1$, respectively). After nasal administration of hNSCs in the normal group, only ++ signals were detected in the liver at $24 \mathrm{~h}$ (positive individuals $=2$ ). In the $\mathrm{NaCl}$ control group, positive signals were detected in one individual. Since the number of positive microdroplets was close to the cutoff value, it could not 

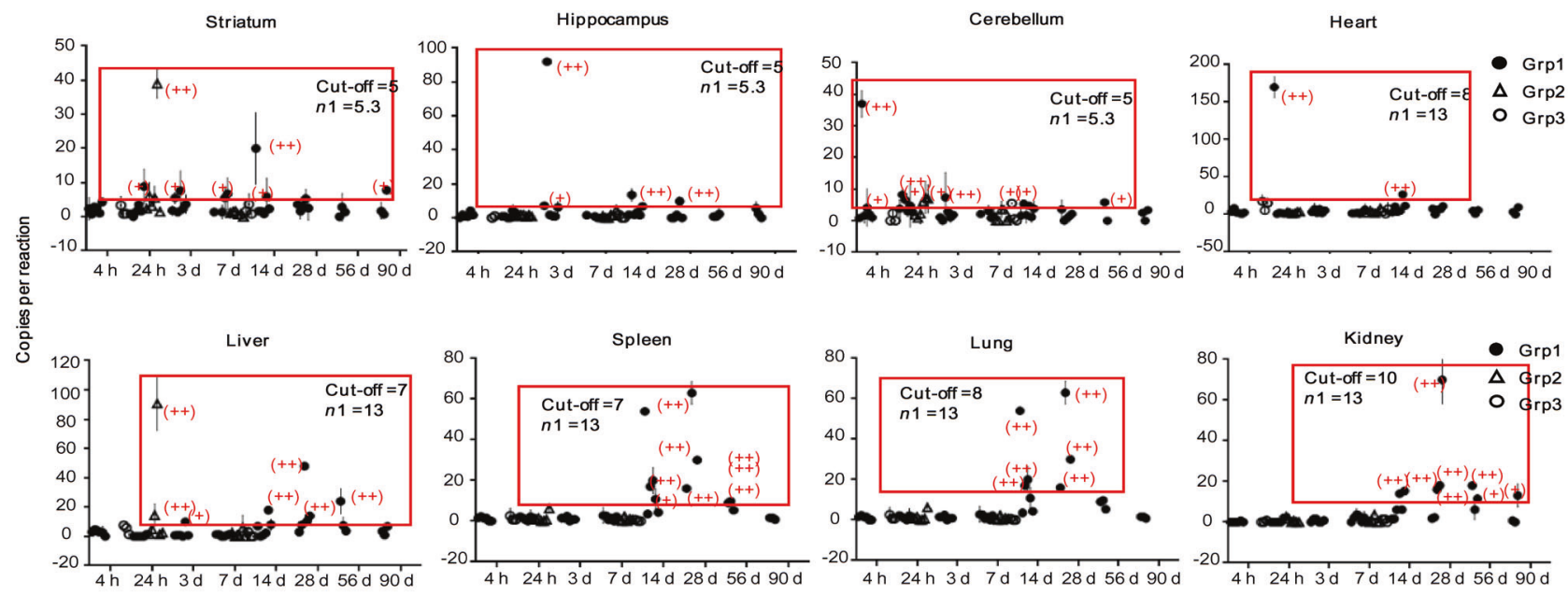

Fig. 6 Biodistribution profile of hNSCs using ddPCR. The hNSC concentration (copies per $20 \mu \mathrm{L} P C R$ )-time curves of mice after intranasal administration of $10^{6}$ cells. Data are shown as the mean \pm SD from two independently analyzed reactions. The copies were derived from the $\sim 20 \mu \mathrm{L}$ (300 ng gDNA) of frozen section tissues (striatum, hippocampus, cerebellum) and $20 \mu \mathrm{L}$ ( $80 \mathrm{ng}$ gDNA) of FFPE section tissues (heart, liver, spleen, lung, kidney). Grp1: MPTP model + hNSC intranasal administration; Grp2: Normal model + hNSC intranasal administration; Grp3: MPTP model $+\mathrm{NaCl}$ intranasal administration

be excluded that this result was statistically a $5 \%$ false positive result (Fig. 6).

In whole blood tissue, + signals were detected only in MPTP mice (positive individuals $=2$ ) at $4 \mathrm{~h}$, and no signals were detected for the other groups.

\section{DISCUSSION}

Tracing stem cell distribution and survival over a long period of time after transplantation would support the in vivo efficacy evaluation. Until now, behavioral tests, noninvasive imaging, and subsequent histological analysis have been the preferred methods to evaluate the viability, differentiation, and effects of transplanted cells. However, sensitivity is still a very large hurdle for tracing NSCs. The present study is the first to comprehensively evaluate the biodistribution of hNSCs in preclinical trials using imaging, $\mathrm{IHC}$ and $\mathrm{ddPCR}$ quantitative tools. The superior sensitivity and higher throughput obtained from ddPCR in comparison to BLI, ${ }^{89} \mathrm{Zr}$-PET/ $\mathrm{CT}$, and IHC warrant its advantage for evaluating NSC biodistribution.

Unlike the hNSC transplantation, in the present study, hNSCs were nasally administered, which led to the traced biodistribution of hNSCs in the brain, which required the high sensitivity of the hNSC detection assay. It was demonstrated that high sensitivity and accurate quantification could be applied to the PK profiling of hNSCs in animal models, such as ddPCR. Although ddPCR does not allow for real-time monitoring in vivo, this disadvantage can be compensated for by the advantages of high throughput and accurate quantification. In contrast to real-time quantitative PCR, the quantitative method of $\mathrm{ddPCR}$ does not depend on the cycle threshold of the amplification curve, so ddPCR is not affected by the amplification efficiency, and it is not necessary to adopt a housekeeping gene and a standard curve [17]. In addition, the dependence on lab technique and experience of ddPCR is relatively low in comparison to IHC and MRI labeling and imaging. According to the guidelines of stem cell therapy PK evaluation [21-24], tracing NSCs, and differentiation is very important for both early and late stages. Correspondingly, another advantage of ddPCR is that for the positive samples in the late stage, different primers targeting different stem biomarker genes can be designed to detect the differentiation status.
This analytical study involves three processes: (a) Sections acquisition: for the current study, four frozen sections or four FFPE sections were used for nucleic acid extraction; limited by the size of the tissue, the sizes of the sections may vary. Selecting four slices is based on the minimum number of tissue sections available in the range that the extraction kit can handle. (b) Nucleic acid measurement and quality control: we selected the purity of the nucleic acid to be not lower than 1.6 as the standard of quality control. The total DNA yielded from FFPE samples varies greatly, depending on the tissue type, as well as the fixation and embedding conditions. (c) Normalization of sample input of ddPCR: in the current study, there are several methods of normalization: (1) quality concentration normalization (for example, copies/ng), or (2) copies/section (for example, copies/ 4 sections) normalization. We used quality concentration normalization in the current study. For the $20 \mu \mathrm{L}$ ddPCR reaction, the maximal template amount is limited, so we normalized the template to the minimal lower threshold, which is $300 \mathrm{ng}$ of gDNA of frozen section tissues and $80 \mathrm{ng}$ of gDNA of FFPE section tissues. For normalization between frozen sections and FFPE sections, because the quality of the tissues was very different, the content of stem cells and extraction efficiency also varied, so we performed the normalization of the frozen sections and FFPE sections separately.

To date, there have not been any preclinical studies reporting stem cell biodistribution in complicated matrices; therefore, in terms of normalization, more scientific and accurate methods need to be explored.

In the current study, we not only performed a PK study for the nasal administration of hNSCs but, more importantly, attempted to understand the mechanism of hNSC therapy. Transplanted NSCs exert their beneficial effects not only through the replacement of damaged cells but also via bystander immunomodulatory and neurotrophic actions [25-27]. Here, although the actual number of brains is very small and no live hNSCs were detected in the brain using immunofluorescence imaging (cell segments, Supplementary Fig. 3) after 14 days, the obvious efficacy in disease animal models in the late stage ( 3 months later) indicated that hNSCs may exert their function through paracrine action instead of directly replacing the damaged neurons. The results were consistent with previous literature [28]. In addition, our data indicate that compared with the normal group, MPTP model mice 
seem to have longer retention of hNSCs in vivo. There are two possible causes: (1) for the mouse MPTP model, striatum dopaminergic neurons are severely compromised, and the dopamine neurotransmitters are significantly diminished. These damaged regions release various chemokines, which may attract stem cells into the brain, which can migrate to the damaged regions to fulfill their regenerative function. (2) The integrity of the blood-brain barrier is jeopardized in MPTP mice, and the impaired BBB may also allow for the better penetration of stem cells into the brain, thus yielding better biodistribution.

In conclusion, the present study is the first to report the application of ddPCR in the pharmacokinetic profile description of hNSC tracking in preclinical studies. For a comparative strategy, we recommend combining high sensitivity ddPCR with other methods to trace hNSCs.

\section{ACKNOWLEDGEMENTS}

This study was supported by the National Thirteen Five Major Special Fund "Nonclinical and Clinical PK, PD, and Immunogenicity Evaluation of Biosimilar" (2015ZX09501008-006) and "Core Technology-based Nonclinical Evaluation of Biological macromolecular drugs" (2015ZX09501007-002).

\section{AUTHOR CONTRIBUTIONS}

JF, ZW and HFS designed the research; FW, ZW, FW, JZ, YJS, CFL, MJX, XC, SW, JWZ, $\mathrm{XFZ}$, and XMW performed the research; FW, FW, KD, JZ, and JF analyzed the data; and ZW, FW, KD, JF, and HFS wrote the paper.

\section{ADDITIONAL INFORMATION}

The online version of this article (https://doi.org/10.1038/s41401-019-0313-x) contains supplementary material, which is available to authorized users.

Competing interests: The authors declare no competing interests.

\section{REFERENCES}

1. Ramos-Gómez M, Martínez-Serrano A. Tracking of iron-labeled human neural stem cells by magnetic resonance imaging in cellreplacement therapy for Parkinson's disease. Neural Regen Res. 2016;11:49-52.

2. Liau MT, Amini F, Ramasamy TS. The therapeutic potential of stem cells and progenitor cells for the treatment of Parkinson's disease. Tissue Eng Regen Med. 2016;13:455-64.

3. Liu Z, Li Z. Molecular imaging in tracking tumor-specific cytotoxic T lymphocytes (CTLs). Theranostics. 2014:4:990-1001.

4. Andrzejewska A, Nowakowski A, Janowski M, Bulte JW, Gilad AA, Walczak P. et al. Pre- and postmortem imaging of transplanted cells. Int J Nanomed. 2015;10:5543-59.

5. Gaudet JM, Ribot EJ, Chen Y, Gilbert KM, Foster PJ. Tracking the fate of stem cell implants with fluorine-19 MRI. PLoS One. 2015;10:e0118544.

6. Asiedu KO, Koyasu S, Szajek LP, Choyke PL, Sato N. Bone marrow cell trafficking analyzed by ${ }^{89} \mathrm{Zr}$-oxine positron emission tomography in a murine transplantation model. Clin Cancer Res. 2017;23:2759-68.

7. Moroz MA, Zhang H, Lee J, Moroz E, Zurita J, Shenke L, et al. Comparative analysis of $\mathrm{T}$ cell imaging with human nuclear reporter genes. J Nucl Med. 2015;56:1055-60.
8. Kallur T, Farr TD, Bohm-Sturm P, Kokaia Z, Hoehn M. Spatio-temporal dynamics, differentiation and viability of human neural stem cells after implantation into neonatal rat brain. Eur J Neurosci. 2011;34:382-93.

9. Song G, Chen M, Zhang Y, Cui L, Qu H, Zheng X, et al. Janus iron oxides ${ }^{@}$ semiconducting polymer nanoparticle tracer for cell tracking by magnetic particle imaging. Nano Lett. 2018;18:182-9.

10. Zheng B, Yu E, Orendorff R, Lu K, Konkle JJ, Tay ZW, et al. Seeing SPIOs directly in vivo with magnetic particle imaging. Mol Imaging Biol. 2017;19:385-90.

11. Nejadnik $H$, Pandit $P$, Lenkov $O$, Lahiji AP, Yerneni $K$, Daldrup-Link HE, et al. Ferumoxytol can be used for quantitative magnetic particle imaging of transplanted stem cells. Mol Imaging Biol. 2019;21:465-72.

12. Zheng B, von See MP, Yu E, Gunel B, Lu K, Vazin T, et al. Quantitative magnetic particle imaging monitors the transplantation, biodistribution, and clearance of stem cells in vivo. Theranostics. 2016;6:291-301.

13. Bossolasco P, Cova L, Levandis G, Diana V, Cerri S, Lambertenghi Deliliers G, et al. Noninvasive near-infrared live imaging of human adult mesenchymal stem cells transplanted in a rodent model of Parkinson's disease. Int J Nanomed. 2012;7:435-47.

14. Ramos-Gomez M, Seiz EG, Martinez-Serrano A. Optimization of the magnetic labeling of human neural stem cells and MRI visualization in the hemiparkinsonian rat brain. J Nanobiotechnol. 2015;13:20.

15. Eamegdool SS, Weible MW 2nd, Pham BT, Hawkett BS, Grieve SM, Chan-ling T, et al. Ultrasmall superparamagnetic iron oxide nanoparticle prelabeling of human neural precursor cells. Biomaterials. 2014;35:5549-64.

16. Schintu N, Frau L, Ibba M, Garau A, Carboni E, Carta AR, et al. Progressive dopaminergic degeneration in the chronic MPTP mouse model of Parkinson's disease. Neurotox Res.2009;16:127-39.

17. Quan PL, Sauzade M, Brouzes E. dPCR: a technology review. Sens (Basel). 2018;18:1271-98.

18. Bernsen MR, Guenoun J, van Tiel ST, Krestin GP. Nanoparticles and clinically applicable cell tracking. Br J Radiol. 2015;88:20150375.

19. Gonzalez R, Garitaonandia I, Poustovoitov M, Abramihina T, McEntire C, Culp B, et al. Neural stem cell derived from human parthenogenetic stem cells engraft and promote recovery in a nonhuman primate model of Parkinson's disease. Cell Transplant. 2016;25:1945-66.

20. Li X, Yang Q, Bai J, Xuan Y, Wang Y. Identification of appropriate reference genes for human mesenchymal stem cell analysis by quantitative real-time PCR. Biotechnol Lett. 2015;37:67-73.

21. China Food and Drug Administration. Technical Guidelines for the Development and Evaluation of Biosimilar Drugs (Trial). Beijing: China Food and Drug Administration; 2015.

22. Guidance for industry bioanalytical method validation. Biopharmaceutics Revision 1. Rockville (MD): US Department of Health and Human Services FDA (CDER) and (CVM); 2013. Docket Number:FDA-2013-D-1020.

23. EMEA. Guideline on bioanalytical method validation. Amsterdam (Netherland): EMEA; 2012.

24. Biological sample quantitative analysis method validation guidelines. In : Pharmacopoeia of People's Republic of China. Part 3. Beijing: Medical Science Press; 2015.

25. Dooves S, van der Knaap MS, Heine VM. Stem cell therapy for white matter disorders: don't forget the microenvironment! J Inherit Metab Dis. 2016;39:513-8.

26. Abati E, Bresolin N, Comi GP, Corti S. Preconditioning and cellular engineering to increase the survival of transplanted neural stem cells for motor neuron disease therapy. Mol Neurobiol. 2019;56:3356-67.

27. Gao L, Xu W, Li T, Chen J, Shao A, Yan F, et al. Stem cell therapy: a promising therapeutic method for intracerebral hemorrhage. Cell Transplant. 2018;27: 1809-24.

28. Zuo F, Xiong F, Wang X, Li X, Wang R, Ge W, et al. Intrastriatal transplantation of human neural stem cells restores the impaired subventricular zone in Parkinsonian mice. Stem Cells. 2017:35:1519-31. 